\title{
Consenso Brasileiro de Espondiloartropatias: Espondilite Anquilosante e Artrite Psoriásica Diagnóstico e Tratamento - Primeira Revisão
}

\section{First Update on the Brazilian Consensus for the Diagnosis and Treatment of Spondyloarthropathies: Ankylosing Spondylitis and Psoriatic Arthritis}

Percival D. Sampaio-Barros ${ }^{(1)}$, Valderílio Feijó Azevedo ${ }^{(2)}$, Rubens Bonfiglioli( ${ }^{(3)}$, Wesley R. Campos ${ }^{(4)}$, Sueli Coelho da Silva Carneiro ${ }^{(5)}$, Marco Antonio P. Carvalho ${ }^{(6)}$, Célio Roberto Gonçalves ${ }^{(7)}$, Maria Odete E. Hilário ${ }^{(8)}$, Mauro W. Keiserman ${ }^{(9)}$, Nocy H. Leite ${ }^{(10)}$, Karen Mallmann ${ }^{(11)}$, Eduardo de Souza Meirelles ${ }^{(12)}$, Walber P. Vieira ${ }^{(13)}$, Antonio Carlos Ximenes ${ }^{(14)}$

Descrição do método e coleta de evidências: Reunião consensual para elaboração do texto com inclusão das citações bibliográficas, numa colaboração de reumatologistas com as especialidades de reumatologia pediátrica, dermatologia, coloproctologia e oftalmologia. Foram convidados 10 reumatologistas responsáveis pelo ambulatório de espondiloartropatias em suas instituições (ou seus representantes); cada participante foi convidado a fazer uma análise crítica, utilizando o conceito da medicina baseada em evidências, de um aspecto diferente dentro do espectro do diagnóstico e tratamento das espondiloartropatias; foi convidado um especialista em cada área para fazer a mesma análise crítica nos tópicos espondiloartropatias juvenis (reumatologista pediátrico), psoríase (dermatologista), doenças inflamatórias intestinais (coloproctologista) e uveite anterior (oftalmologista). Após a análise do Consenso Brasileiro de Espondiloartropatias (2004) como texto-base de apoio para as discussões, todos os especialistas se reuniram no período entre 4 e 5 de maio de 2007 , a fim de discutir individualmente cada um dos 14 tópicos elaborados e estabelecer um consenso baseado em evidências, a partir do qual o coordenador redigiu o texto do consenso, submetido à apreciação de todos os participantes para a realização dos ajustes finais.

1. Assistente-doutor da Disciplina de Reumatologia do Departamento de Clínica Médica da Faculdade de Ciências Médicas da Universidade Estadual de Campinas (FCM-UNICAMP). Presidente da Comissão de Espondiloartropatias da Sociedade Brasileira de Reumatologia (SBR).

2. Professor Assistente da Disciplina de Reumatologia da Universidade Federal do Paraná (UFPR). Mestre em Medicina Interna

3. Professor Assistente da Disciplina de Reumatologia da Pontifícia Universidade Católica de Campinas (PUCCAMP).

4. Professor Adjunto, Doutor em Oftalmologia da Universidade Federal de Minas Gerais (UFMG).

5. Professora Adjunta da Faculdade de Ciências Médicas da Universidade Estadual do Rio de Janeiro (UERJ) e Professora do Programa de Pós-Graduação em Medicina da Universidade Federal do Rio de Janeiro (UFRJ).

6. Professor Adjunto, Doutor de Reumatologia do Departamento do Aparelho Locomotor da Universidade Federal de Minas Gerais (UFMG).

7. Professor Doutor-Assistente e Coordenador da Unidade de Espondiloartropatias da Disciplina de Reumatologia da Faculdade de Medicina da Universidade de São Paulo (FMUSP)

8. Professora Associada e Responsável pelo Setor de Reumatologia Pediátrica da Universidade Federal de São Paulo (UNIFESP).

9. Professor Regente da Disciplina de Reumatologia da Faculdade de Medicina da Pontifícia Universidade Católica do Rio Grande do Sul (PUC-RS).

10. Professor Titular de Reumatologia da Faculdade de Medicina Souza Marques, Rio de Janeiro - RJ.

11. Professora da Disciplina de Coloproctologia da Fundação Faculdade Federal de Ciências Médicas de Porto Alegre (FFFCMPA)

12. Assistente-Doutor e Chefe do Grupo de Reumatologia do Instituto de Ortopedia e Traumatologia da FMUSP.

13. Chefe do Serviço de Reumatologia e Coordenador da Residência Médica do Hospital Geral de Fortaleza.

14. Chefe do Departamento de Medicina Interna do Hospital Geral de Goiânia. Doutor em Reumatologia pela FMUSP

Conflitos de interesse: (1) Recebimento de honorários por conferência ou palestra, bem como financiamento para realização de pesquisa, organização de atividade de ensino ou comparecimento a simpósios e congressos: Abbott, Actelion, Bristol-Myers-Squibb, Mantecorp, Merck Sharp E Dohme, Pfizer, Roche e Wyeth; (2) Auxílio para viagens e recebimento de honorários por palestras: Abbott e Mantecorp; (5) Auxílio para viagens e comparecimento a simpósios e congressos: Mantecorp e Wyeth; (7) Auxílio para participação em congresso: Abbott; (9) Recebimento de honorários por conferência ou palestra, bem como financiamento para realização de pesquisa, organização de atividade de ensino ou comparecimento a simpósios e congressos: Abbott e Bristol-Myers-Squibb; ( 12 ) Recebimento de honorários por conferência ou palestra: Abbott e Mantecorp; (14) Recebimento de honorários por conferência ou palestra, bem como financiamento para realização de pesquisa, organização de atividade de ensino ou comparecimento a simpósios e congressos: Abbott, Bristol-Myers-Squibb, Mantecorp, Merck Sharp E Dohme, Pfizer, Roche e Wyeth. (3), (4), (6), (8), (10), (11) e (13) declararam não ter conflito de interesse.

Endereço para correspondência: Dr. Percival D. Sampaio-Barros. Av. Brigadeiro Luiz Antonio, 2466 - conjs. 93-94, CEP 01402-000. São Paulo, SP, Brasil, e-mail: sbre@terra.com.br 
Objetivo: Oferecer informações sobre a otimização do diagnóstico e do tratamento das diferentes espondiloartropatias.

Grau de recomendação e força de evidência científica:

A: Grandes ensaios clínicos aleatorizados e metaanálises.

B: Estudos clínicos e observacionais bem desenhados.

C: Relatos e séries de casos clínicos.

D: Publicações baseadas em consensos e opiniões de especialistas.

\section{INTRODUÇÃO}

O conceito das espondiloartropatias soronegativas foi estabelecido em 1974, quando os pesquisadores ingleses Moll e Wright propuseram que se englobasse dentro de um mesmo conjunto algumas doenças até então consideradas completamente distintas entre si, mas que, na verdade, apresentavam diversas características comuns. Tais características englobavam aspectos clínicos (dor axial inflamatória, associada à artrite, predominante em grandes articulações de membros inferiores, e entesopatias periféricas), radiológicos (sacroiliíte) e laboratoriais (soronegatividade para o fator reumatóide, pois, até a década de 1970, alguns pesquisadores consideravam a espondilite anquilosante como o componente axial da artrite reumatóide) em indivíduos com predisposição genética (ligada ao antígeno de histocompatibilidade HLA-B27). Este conjunto incluiu a espondilite anquilosante, a artrite psoriásica, a artrite reativa e a síndrome de Reiter, e as artropatias enteropáticas

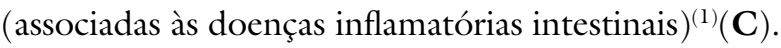

Nos últimos 20 anos, em virtude do crescente número de pacientes que apresentam um diagnóstico provável, mas que não conseguem preencher os critérios diagnósticos para uma doença definida dentro do grupo, criou-se o conceito das espondiloartropatias indiferenciadas. Recentemente, especialistas internacionais propuseram a mudança de nome para espondiloartropatias, suprimindo o termo soronegativas $^{(2)}(\mathbf{C})$.

\section{CONSIDERAÇÕES GERAIS SOBRE A ABOR- DAGEM DIAGNÓSTICA DE PACIENTES COM ESPONDILOARTROPATIAS}

Cada uma das doenças definidas dentro do grupo (espondilite anquilosante, artrite psoriásica, artrite reativa e artrite enteropática) tem seus próprios critérios diagnós- ticos. No entanto, existem muitos casos ditos indiferenciados ou incompletos. Na tentativa de conseguir englobar todo este amplo e heterogêneo grupo de pacientes, vários critérios classificatórios foram propostos. Analisando-os, observa-se que os critérios do Grupo Europen de Estudo das Espondiloartropatias ${ }^{(3)}(\mathbf{D})$ (Tabela 1) são os mais utilizados na prática clínica diária, sendo facilmente lembrados e aplicados; para ser incluído dentro do grupo é necessário preencher pelo menos um critério maior e um menor.

TABELA 1

CRITÉRIOS CLASSIFICATÓRIOS DE ESPONDILOARTROPATIAS Grupo Europeu de Estudo das Espondiloartropatias (ESSG), 1991

\begin{tabular}{l} 
Dor axial inflamatória ou $\begin{array}{l}\text { Sinovite assimétrica predominante em } \\
\text { membros inferiores }\end{array}$ \\
Pelo menos um dos seguintes critérios: \\
\hline $\begin{array}{l}\text { História familiar positiva (espondilite anquilosante, psoríase, uveíte anterior, } \\
\text { doença inflamatória intestinal) }\end{array}$ \\
Psoríase cutânea \\
Doença inflamatória intestinal \\
Uretrite ou diarréia aguda até 4 semanas precedendo a artrite \\
Dor em nádegas alternante \\
Entesopatia (inserção do tendão de Aquiles ou fáscia plantar) \\
Sacroiliíte (bilateral graus 2 a 4 ou unilateral graus 3 ou 4) \\
\hline
\end{tabular}

Extraída da referência 3.

Sensibilidade: $86 \%$; especificidade: $87 \%$.

\section{CONSIDERAÇÕES GERAIS SOBRE A ABOR- DAGEM TERAPÊUTICA DE PACIENTES COM ESPONDILOARTROPATIAS}

As doenças crônicas, como as espondiloartropatias, na maioria das vezes causam um sentimento de desamparo e inutilidade mais assustador do que a doença em si. Por isso, deve-se sempre informar o paciente de forma realista sobre as perspectivas prognósticas de sua doença, perspectivas essas que são freqüentemente otimistas, desde que haja uma abordagem global de atendimento. O paciente deve estar absolutamente ciente de que sua participação - especialmente no estar bem consigo mesmo, apesar da doença - será um ponto de partida fundamental para o sucesso terapêutico.

Deve-se realizar o seguimento dos pacientes e o tratamento de forma individual, caso a caso, já que a história natural da doença pode cursar com flutuações durante seu curso evolutivo. O médico deve considerar o impacto da doença para o indivíduo dentro de sua comunidade, com as 
devidas implicações no seu ambiente familiar e de trabalho, além dos aspectos sociais e financeiros. Apesar de inexistir, até o momento, um tratamento específico para a cura, deve-se ressaltar que os recursos terapêuticos disponíveis possibilitam, de um modo geral, um adequado controle da doença. Um ponto fundamental para tais conquistas está centrado numa boa relação médico-paciente; quando necessário, o médico assistente deve indicar auxílio psicoterápico e/ou uso de drogas antidepressivas.

\section{ESPONDILITE ANQUILOSANTE}

A espondilite anquilosante (EA) é uma doença inflamatória crônica que acomete preferencialmente a coluna vertebral, podendo evoluir com rigidez e limitação funcional progressiva do esqueleto axial. Geralmente se inicia no adulto jovem $\left(2^{a}\right.$ a $4^{a}$ décadas da vida), preferencialmente do sexo masculino, da cor branca e em indivíduos HLAB27 $\operatorname{positivos}^{(4)}(\mathbf{D})$.

A EA de início no adulto, que se inicia a partir dos 16 anos, costuma ter como sintoma inicial a lombalgia de ritmo inflamatório, com rigidez matinal prolongada e predomínio dos sintomas axiais durante sua evolução. A EA juvenil, que se manifesta antes dos 16 anos de idade, costuma iniciar-se com artrite e entesopatias periféricas, evoluindo, somente após alguns anos, com a característica lombalgia de ritmo inflamatório. A EA juvenil costuma ter diagnóstico mais tardio, e muitos destes pacientes podem ser diagnosticados como artrite idiopática juvenil no início dos sintomas. O comprometimento do quadril é mais freqüente na criança do que no adulto, o que determina um pior prognóstico, pela necessidade de próteses totais de quadril em muitos pacientes ${ }^{(5)}(\mathbf{B})$.

A freqüente associação com o HLA-B27 faz que a EA seja mais comum em populações brancas, em que a prevalência do HLA-B27 é significativamente maior. Por sua vez, a positividade do HLA-B27 nos pacientes espondilíticos pode variar entre $80 \%$ e $98 \%$, sendo mais elevada em populações brancas não miscigenadas do norte da Europa ${ }^{(6)}$ (D). Em virtude da extrema raridade da presença do HLAB27 em populações negras africanas, a EA é muito pouco freqüente nessa etnia; no Brasil, país de intensa miscigenação étnica, a EA, bem como outras espondiloartropatias, costuma ser encontrada em mulatos (devido à influência da ascendência genética branca), mas é bastante rara em negros não miscigenados ${ }^{(7)}(\mathbf{B})$.

Inicialmente, o paciente espondilítico costuma queixarse de dor lombar baixa de ritmo inflamatório, caracterizada por melhorar com o movimento e piorar com o repouso, apresentando rigidez matinal prolongada. A evolução costuma ser ascendente, acometendo progressivamente a coluna dorsal e cervical, contribuindo para o desenvolvimento da "postura do esquiador", caracterizada pela retificação da lordose lombar, acentuação da cifose dorsal e retificação da lordose cervical (com projeção da cabeça para a frente). $\mathrm{O}$ acometimento articular periférico caracteriza-se pela presença de oligoartrite e entesopatias. A oligoartrite predomina em grandes articulações de membros inferiores, como tornozelos, joelhos e coxofemorais. As entesopatias (inflamações nas inserções dos tendões e/ou ligamentos nos ossos) costumam ser manifestações iniciais na EA de início juvenil e acometem preferencialmente a inserção de tendão de Aquiles e a fáscia plantar. Quanto às manifestações extra-articulares, a mais freqüente é a uveíte anterior, aguda, unilateral, recorrente, que se pode observar em até $40 \%$ dos pacientes num seguimento prolongado, estando geralmente associada ao HLA-B27 positivo e que raramente cursa com seqüelas $^{(8)}(\mathbf{D})$.

\section{Diagnóstico}

Para a confirmação diagnóstica da EA, os critérios mais utilizados são os de Nova York modificados, que combinam critérios clínicos e radiográficos. Os critérios clínicos são: 1) Dor lombar de mais de três meses de duração que melhora com o exercício e não é aliviada pelo repouso; 2) Limitação da coluna lombar nos planos frontal e sagital; 3 ) Expansibilidade torácica diminuída (corrigida para idade e sexo). Os critérios radiográficos são: 1) Sacroiliíte bilateral, grau 2,3 ou $4 ; 2$ ) Sacroiliíte unilateral, grau 3 ou 4 . Para o diagnóstico de EA é necessária a presença de um critério clínico e um critério radiográfico ${ }^{(9)}(\mathbf{C})$.

Nos últimos anos, em virtude do surgimento de drogas mais eficazes para o tratamento da EA, múltiplos instrumentos de avaliação clínica $^{(10)}(\mathbf{D})$ e de imagem $^{(11)}(\mathbf{D})$ foram propostos. Entre estes instrumentos, podemos citar um índice de atividade de doença, o BASDAI (Bath Ankylosing Spondylitis Disease Activity Index); índices funcionais, como o BASFI (Bath Ankylosing Spondylitis Functional Index) e o índice funcional de Dougados; um índice metrológico, o BASMI (Bath Ankylosing Spondylitis Metrology Index); índices radiológicos, como o BASRI (Bath Ankylosing Spondylitis Radiology Index) e o mSASSS (modified Stokes Ankylosing Spondylitis Scoring System); e indicadores de qualidade de vida, como o ASQoL (Ankylosing Spondylitis Quality of Life) e o HAQ-S (Health Assessment Questionnaire for Spondyloarthropathies). 
Entre todos estes índices, o BASDAI ${ }^{(12)}(\mathbf{D})$, agora validado para o português ${ }^{(13)}(\mathbf{D})$ (Tabela 2), é considerado o melhor índice para acompanhar periodicamente a evolução do tratamento da EA na prática clínica diária, por refletir um conjunto de situações que podem modificar-se com o tratamento, como a sensação de fadiga, a dor axial inflamatória, o componente periférico e a intensidade e duração da rigidez matinal. O BASFI ${ }^{(14)}(\mathbf{D})$, também validado para o português $^{(13)}(\mathbf{D})$ (Tabela 3), é bastante eficiente para avaliar o grau de incapacidade funcional dos pacientes, porém apresenta pequena variabilidade, a despeito do tratamento, em casos com longa evolução de doença, onde muitas vezes algumas alterações funcionais não são reversíveis. Recentemente foi realizada a tradução para a língua portuguesa, validação e adaptação transcultural do HAQ-S, um questionário auto-aplicativo para avaliar qualidade de vida, devendo ser mais uma opção a ser utilizada para avaliação clínica dos pacientes com $\mathrm{EA}^{(15)}(\mathbf{D})$.

TABELA 2

BASDAI, VALIDADO PARA O PORTUGUÊS

\begin{tabular}{|c|c|}
\hline $\begin{array}{l}\text { Coloque uma ma } \\
\text { cada questão rel }\end{array}$ & resposta para \\
\hline 1. Como você des & ocê tem tido? \\
\hline 0 & $10 \mathrm{~cm}$ \\
\hline Nenhum & Intenso \\
\hline $\begin{array}{l}\text { 2. Como você des } \\
\text { quadril relacionad }\end{array}$ & Is costas e no \\
\hline 0 & $10 \mathrm{~cm}$ \\
\hline Nenhum & Intenso \\
\hline $\begin{array}{l}\text { 3. Como você des } \\
\text { articulações sem }\end{array}$ & aço) nas outras \\
\hline 0 & $10 \mathrm{~cm}$ \\
\hline Nenhum & Intenso \\
\hline
\end{tabular}

4. Como você descreveria o grau total de desconforto que você teve ao toque ou à compressão em regiões do corpo doloridas?

\begin{tabular}{ll}
0 & $10 \mathrm{~cm}$ \\
\hline Nenhum & Intenso
\end{tabular}

5. Como você descreveria a intensidade da rigidez matinal que você tem tido a partir da hora em que você acorda?

\begin{tabular}{ll}
0 & $10 \mathrm{~cm}$ \\
\hline Nenhum & Intenso
\end{tabular}

6. Quanto tempo dura sua rigidez matinal a partir do momento em que você acorda?

0

$30 \min$

$1 \mathrm{~h}$

$1 \mathrm{~h} 30$

$2 \mathrm{~h}$

BASDAI: soma dos valores das questões 1, 2, 3, 4 e a média dos valores da 5 e 6 , dividindo este total por 5 .

Extraída da referência 13 .
TABELA 3

BASFI, VALIDADO PARA O PORTUGUÊS

Faça uma marca em cada linha abaixo de cada pergunta, indicando o seu grau de capacidade para realizar as seguintes atividades durante a última semana

1. Vestir meias ou meia-calça sem ajuda ou auxílio de aparelhos.

$\begin{array}{ll}0 & 10 \mathrm{~cm} \\ \text { Fácil } & \text { Impossivel }\end{array}$

2. Curvar o corpo da cintura para cima para pegar uma caneta no chão sem o uso de um instrumento de auxílio.

\begin{tabular}{ll}
0 & $10 \mathrm{~cm}$ \\
\hline Fácil & Impossivel
\end{tabular}

3. Alcançar uma prateleira alta sem ajuda ou auxílio de um instrumento.

\begin{tabular}{ll}
0 & $10 \mathrm{~cm}$ \\
\hline Fácil & Impossível
\end{tabular}

4. Levantar-se de uma cadeira sem braços da sala de jantar sem usar as mãos ou qualquer outro tipo de ajuda.

$\begin{array}{ll}0 & 10 \mathrm{~cm} \\ \text { Fácil } & \text { Impossível }\end{array}$

5. Levantar-se quando deitado de costas no chão sem ajuda.

\begin{tabular}{ll}
0 & $10 \mathrm{~cm}$ \\
\hline Fácil & Impossivel
\end{tabular}

6. Ficar em pé sem ajuda por 10 minutos sem desconforto

$\begin{array}{ll}0 & 10 \mathrm{~cm} \\ \text { Fácil } & \text { Impossível }\end{array}$

7. Subir 12 a 15 degraus sem usar o corrimão ou outra forma de apoio (andador); um pé em cada degrau.

$\begin{array}{ll}0 & 10 \mathrm{~cm} \\ \text { Fácil } & \text { Impossível }\end{array}$

8. Olhar para trás, virando a cabeça sobre o ombro sem virar o corpo.

\begin{tabular}{ll}
0 & $10 \mathrm{~cm}$ \\
\hline Fácil & Impossivel
\end{tabular}

9. Realizar atividades que exijam esforço físico, isto é, fisioterapia, jardinagem ou esporte.

\begin{tabular}{ll}
0 & $10 \mathrm{~cm}$ \\
\hline Fácil & Impossivel
\end{tabular}

10. Ter um dia repleto de atividades, seja em casa ou no trabalho.

$\begin{array}{ll}0 & 10 \mathrm{~cm} \\ \text { Fácil } & \text { Impossível }\end{array}$

BASFI: Somatório dos valores em $\mathrm{cm}$ anotados nas EVA é dividido por 10 e dado o valor final.

Extraída da referência 13.

\section{TRATAMENTO}

Inicialmente, o paciente espondilítico deve ser informado que, embora a EA seja uma doença crônica, apresenta boas perspectivas terapêuticas atualmente, e que somente se costuma observar a agregação familiar da doença em famílias de pacientes HLA-B27 positivo. O amparo psicológico, 
visando à perfeita integração à sociedade, é fundamental na condução terapêutica dos pacientes espondilíticos (ver item Considerações gerais sobre a abordagem terapêutica de pacientes com espondiloartropatias).

Deve-se realizar a fisioterapia, notadamente os programas de exercícios supervisionados, de maneira sistemática em todos os estágios da doença, já que os seus benefícios na prevenção de limitações funcionais e na restauração de uma adequada mobilidade articular somente são observados no período em que o paciente os realiza ${ }^{(16)}(\mathbf{A})$. Embora se observe uma significativa heterogeneidade nos objetivos dos trabalhos envolvendo fisioterapia na EA, a cinesioterapia tem oferecido melhores resultados, tendo os meios físicos representado papel coadjuvante.

Devem-se utilizar os antiinflamatórios não hormonais (AINHs) desde o início do tratamento; não existem trabalhos evidenciando que um determinado AINH se mostre superior aos outros na comparação $\operatorname{direta}^{(17)}(\mathbf{D})$. O uso dos antiinflamatórios COX-2 preferenciais e específicos, como o etoricoxibe ${ }^{(18)}(\mathbf{A})$ e o celecoxibe ${ }^{(19)}(\mathbf{A})$, em pacientes com potencial risco de toxicidade gastrointestinal ou que não toleram os AINHs convencionais, parece representar uma boa opção para casos de uso prolongado de AINH. Estudo recente demonstra que o uso contínuo dos AINHs apresenta melhor resposta quanto aos danos estruturais da doença, em comparação ao uso intermitente dos mesmos ${ }^{(20)}$ (A). Ainda não se estabeleceu por quanto tempo se deve utilizar o AINH de forma contínua nos pacientes com EA, mas poder-se-ia considerar que sua retirada seja lenta e gradual, após a completa remissão clínica e laboratorial da enfermidade ${ }^{(21)}(\mathbf{D})$.

Reserva-se o uso de corticosteróides a casos específicos. Em pacientes com artrite periférica persistente, o uso de prednisona, até a dose de $10 \mathrm{mg} /$ dia (ou equivalente) deve ser intermitente, enquanto houver atividade de doença $a^{(22)}$ (D). Pode-se usar a metilprednisolona por via endovenosa em casos muito sintomáticos ${ }^{(23)}(\mathbf{A})$. O uso de corticosteróide por via intra-articular pode ser uma alternativa em casos de artrite persistente ou sacroiliíte refratária (nestes casos, recomenda-se que a infiltração seja guiada por tomografia computadorizada ou ressonância magnética $)^{(24)}(\mathbf{C})$. É importante salientar que se deve evitar o uso dos corticosteróides a longo prazo nos pacientes espondilíticos, pois predispõe à desmineralização óssea, já que se observa baixa densidade mineral óssea ${ }^{(25)}(\mathbf{B})$ e predisposição às fraturas vertebrais $^{(26)}(\mathbf{B})$ na EA mesmo em seus estágios iniciais.

O uso de fármacos de ação lenta indutores de remissão para o tratamento da doença axial na EA tem sido bastante desapontador, embora se obtenham alguns resultados com a doença periférica. Nos pacientes que não responderam a, no mínimo 2 AINHs, no período mínimo de observação de 3 meses, e que apresentam envolvimento articular periférico proximal (quadris e ombros) ou distal (demais articulações periféricas), pode-se utilizar drogas de base de ação prolongada ${ }^{(27)}(\mathbf{D})$. A sulfasalazina, na dose de 30 a $50 \mathrm{mg} / \mathrm{kg} /$ dia, apresenta resposta mais significativa na artrite periférica $^{(28)}(\mathbf{B})$ e na prevenção de surtos recorrentes de uveíte ${ }^{(29)}(\mathbf{A})$. Segundo a Fundação Cochrane, em revisão sistemática que envolveu 11 ensaios randomizados, pacientes com doença precoce, níveis elevados de VHS (doença mais ativa) e artrite periférica, podem se beneficiar do uso da sulfasalazina ${ }^{(30)}(\mathbf{A})$. O metotrexato, na dose de 7,5 a $25 \mathrm{mg}$ semanal, por via oral ou intramuscular, também apresenta melhor resposta nos pacientes com EA com comprometimento periférico $^{(31)}(\mathbf{B})$. Revisão sistemática da Fundação Cochrane, que envolveu apenas três ensaios randomizados, não encontrou evidências para o emprego do MTX na forma axial da EA, questionando sua indicação, até mesmo no envolvimento periférico da doença ${ }^{(32)}(\mathbf{A})$. Quanto à leflunomida, houve ausência de eficácia, mesmo na doença periférica ${ }^{(33)}(\mathbf{D})$. Embora os estudos iniciais com a talidomida ${ }^{(34)}(\mathbf{B})$ e com o pamidronato ${ }^{(35)}(\mathbf{A})$ tenham apresentado resultados promissores (estudos isolados $\mathrm{e}$ com pequenas casuísticas), ainda serão necessários outros estudos para estabelecer os reais riscos e benefícios de seu uso a médio e longo $\operatorname{prazo}^{(33)}(\mathbf{D})$.

Atualmente, existem evidências consistentes de que os agentes biológicos dirigidos contra o fator de necrose tumoral alfa $(\mathrm{TNF} \alpha)$ representam uma boa opção terapêutica nos pacientes com intensa atividade de doença na EA. Os atuais medicamentos anti-TNF $\alpha$ com uso aprovado para o tratamento da EA são o infliximabe, o etanercepte e o adalimumabe. O infliximabe é uma medicação de uso endovenoso, que requer que sua administração seja realizada em centros de infusão que tenham equipe médica com experiência no acompanhamento destes casos; recomenda-se sua administração na dose de 3 a $5 \mathrm{mg} / \mathrm{kg}$, apresentando um esquema de ataque (doses a 0,2 e 6 semanas) e outro de manutenção, a cada 6 a 8 semanas, sendo efetivo no controle da atividade da doença ${ }^{(36)}(\mathbf{A})$, na redução dos índices laboratoriais ${ }^{(37)}(\mathbf{B})$, na redução da inflamação axial detectada na ressonância nuclear magnética ${ }^{(38)}(\mathbf{A})$, na melhoria da qualidade de $\operatorname{vida}^{(39)}(\mathbf{A})$, na redução do uso de $\operatorname{AINH}^{(40)}(\mathbf{B})$ e na redução da incidência de uveíte anterior ${ }^{(41)}(\mathbf{A})$. Estudo multicêntrico alemão mostrou persistência de uma boa resposta clínica no uso por três 
anos de infliximabe ${ }^{(42)}(\mathbf{A})$, mas recidiva após a suspensão abrupta da medicação ${ }^{(43)}(\mathbf{A})$, com nova boa resposta após readministração da medicação ${ }^{(44)}(\mathbf{A})$.

O etanercepte é utilizado na dose de $25 \mathrm{mg}$, via subcutânea, duas vezes por semana, sendo efetivo na melhora da dor e função articulares e na qualidade $\operatorname{de~} \operatorname{vida}^{(45)}(\mathbf{A})$, na redução dos índices laboratoriais ${ }^{(46)}(\mathbf{A})$ e de biomarcadores de degradação cartilaginosa ${ }^{(47)}(\mathbf{B})$, além de diminuir a inflamação axial detectada na ressonância nuclear magnética ${ }^{(48)}$ (A) e a incidência de uveíte anterior ${ }^{(49)}(\mathbf{A})$. Também apresenta eficácia sustentada no uso prolongado ${ }^{(49)}(\mathbf{A})$. Estudo multicêntrico recente revelou que a dose semanal de $50 \mathrm{mg}$ tem eficácia semelhante à dose de $25 \mathrm{mg}$ duas vezes por semana ${ }^{(50)}(\mathbf{B})$. Recentemente, aprovou-se o adalimumabe, na dose de $40 \mathrm{mg}$, por via subcutânea, a cada duas semanas, para uso na EA, apresentando melhora nos parâmetros clínicos $^{(51)}(\mathbf{A})$ e de imagem ${ }^{(52)}(\mathbf{A})$.

A análise da vasta bibliografia publicada nos últimos anos com respeito ao uso dos agentes anti-TNF $\alpha$ na EA demonstra que apresentam eficácia sustentada a curto, médio e longo prazos, mas podem levar à recidiva se suspensos abruptamente, o que subentende um uso por tempo prolongado com diminuição gradativa das doses. Devem ser utilizados como monoterapia, não existindo evidências de uso sinérgico com outras drogas modificadoras de doença, como o metotrexato e a sulfasalazina ${ }^{(53)}(\mathbf{D})$.

Em um estudo recente, o grupo espanhol BIOBADASER demonstrou que, num conjunto de 5.530 pacientes em uso de drogas anti-TNF $\alpha$, a persistência do uso destas medicações a curto e médio prazo é maior nos pacientes com espondiloartropatias que com artrite reumatóide ${ }^{(54)}$ (A). Pode se considerar curta duração de doença, idade mais jovem, PCR elevada, BASFI baixo e BASDAI elevado fatores preditivos de uma resposta clínica significativa em pacientes com $\mathrm{EA}^{(55)}(\mathbf{A})$. E a não-resposta clínica após período mínimo de 12 semanas de uso de agente anti-TNF $\alpha$ parece prever ausência de resposta a longo $\operatorname{prazo}^{(53)}(\mathbf{D})$. A não-resposta a um agente anti-TNF $\alpha$ específico não implica em não resposta à troca de agente biológico ${ }^{(56)}(\mathbf{B})$. Nenhum medicamento anti-TNF $\alpha$ específico mostrou-se superior aos demais.

É importante frisar que o uso destas drogas deve obedecer a rigorosos critérios de indicação e monitoração, visando diminuir os potenciais efeitos colaterais e racionalizar os custos de tratamento ${ }^{(53)}(\mathbf{D})$. Após análise das recomendações para o uso de agentes biológicos nas espondiloartropatias, podemos ratificar os seguintes critérios: indica-se o uso dos agentes anti-TNF $\alpha$ para a redução de sinais e sintomas de pacientes com EA ativa de moderada a grave intensidade em indivíduos com resposta inadequada a dois ou mais AINHs num período mínimo de observação de três meses, e que não responderam à associação com metotrexato ou sulfasalazina, por período adicional de 3 meses, em casos de artrite periférica em atividade. Pode-se considerar EA em atividade quando o paciente mantém BASDAI $\geq 4$ (ver Tabela 2 ).

\section{ARTRITE PSORIÁSICA}

A artrite psoriásica é uma artrite inflamatória, soronegativa para o fator reumatóide, associada à psoríase cutânea ${ }^{(57)}$ (D). A psoríase cutânea é doença bastante freqüente, podendo acometer até 2 a $3 \%$ da população ${ }^{(58)}(\mathbf{D})$; a lesão mais característica é a placa eritêmato-escamosa de bordas bem definidas, que varia em número e em tamanho, estando presente particularmente sobre as superfícies extensoras dos membros e do couro cabeludo ${ }^{(59)}(\mathbf{D})$.

Estatísticas têm demonstrado que 6 a $42 \%$ dos pacientes com psoríase desenvolvem algum tipo de acometimento $\operatorname{articular}^{(58)}(\mathbf{D})$. É mais prevalente em populações brancas. Não costuma ter predomínio de sexo, exceto em subtipos específicos, com predomínio do sexo feminino na forma poliarticular simétrica e do sexo masculino na forma espondilítica. $\mathrm{O}$ acometimento da pele costuma preceder a artrite em 75\% dos casos, havendo início simultâneo em 10\% dos pacientes; nos outros 15\%, a artrite pode preceder a lesão de pele. Não é comum haver correlação entre o tipo ou a gravidade da lesão cutânea e a presença, tipo ou extensão do quadro $\operatorname{articular}^{(60)}(\mathbf{D})$.

A etiopatogenia da artrite psoriásica é multifatorial, e fatores genéticos, ambientais e imunológicos atuam e interagem para o aparecimento da doença ${ }^{(61)}(\mathbf{D})$. Acredita-se que, num indivíduo geneticamente predisposto, a presença de um fator ambiental possa funcionar como "gatilho" para desencadear as alterações imunológicas que darão origem à doença. $\mathrm{O}$ polimorfismo genético pode influenciar esta susceptibilidade; polimorfismo do TNF $\alpha$ tem sido associado com artrite psoriásica e com a presença e progressão de artrite erosiva $^{(62)}(\mathbf{B})$. Entre os fatores ambientais, podemos citar infecção (retrovírus ou bactérias Gram-positivas, como o estreptococo, e mais recentemente o HIV), trauma articular (principalmente em crianças) e algumas drogas (como beta-bloqueadores, lítio, inibidores da enzima conversora da angiotensina e inibidores da COX-1). A psoríase e a artrite psoriásica compartilham os mesmos fatores desencadeantes ambientais e psico afetivos, porém os mecanismos 
neuro-imuno-endócrinos envolvidos neste processo ainda necessitam ser esclarecidos ${ }^{(63)}(\mathbf{D})$.

Do ponto de vista imunológico, observam-se alterações tanto da imunidade humoral quanto da imunidade celular. A pele, as articulações e as ênteses compartilham mecanismos patogênicos semelhantes. Infiltrado composto de células $\mathrm{T}$ ativadas está localizado nas papilas dérmicas, na camada subsinovial e nas ênteses. Outras células envolvidas são as células dendríticas, os macrófagos e as células B. Todas liberam citocinas pró-inflamatórias que levam à ativação de outras células patogênicas, promovem a angiogênese e a reabsorção óssea ${ }^{(64)}(\mathbf{D})$.

\section{Diagnóstico}

Classicamente, a artrite psoriásica apresenta cinco formas clínicas: 1) Oligoartrite assimétrica (70\%): é a forma clínica mais freqüente, acometendo grandes e/ou pequenas articulações; tenossinovites são comuns, caracterizando os "dedos em salsicha"; 2) Poliartrite simétrica (15\%): apresenta quadro articular muito semelhante à artrite reumatóide; pode acometer as articulações interfalangeanas distais, comumente não afetadas na artrite reumatóide; 3 ) Distal (5\%): acomete exclusivamente as articulações interfalangeanas distais, geralmente associada a lesões ungueais ("unha em dedal"); 4) Artrite mutilante (<5\%): é a forma clínica menos freqüente e mais grave, acometendo geralmente indivíduos jovens, na $2^{\text {a }}$ e $3^{\text {a }}$ décadas de vida; afeta as pequenas articulações das mãos e dos pés, evoluindo para deformidades importantes, com encurtamento dos dedos; 5) Espondilite (5\%): os sintomas clínicos costumam ser indistinguíveis daqueles apresentados pela espondilite anquilosante $^{(57)}(\mathbf{D})$.

Nas últimas décadas, foram propostos diversos critérios classificatórios para a artrite psoriásica. Os critérios do Grupo Europeu de Estudo das Espondiloartropatias $(1991)^{(3)}(\mathbf{D})$ (Tabela 1) englobam preferencialmente as formas oligoarticulares e espondilíticas. Recentemente, o Grupo CASPAR (Classification Criteria for Psoriatic Arthritis) propôs novos critérios tentando englobar todo o amplo espectro de casos de artrite psoriásica (Tabela 4) ${ }^{(65)}(\mathbf{D})$. Alguns autores costumam pleitear a inclusão da Síndrome SAPHO (acrônimo de Sinovite, Acne, Pustulose, Hiperostose, Osteíte) como um subtipo distinto da artrite psoriásica; no entanto, o assunto está longe de ser um consenso, aliado à extrema raridade da síndrome em nosso país ${ }^{(66)}(\mathbf{D})$.
TABELA 4

CRITÉRIOS CLASSIFICATÓRIOS DE ARTRITE PSORIÁSICA GRUPO CASPAR, 2006

\begin{tabular}{ll}
\hline $\begin{array}{l}\text { Doença articular inflamatória estabelecida } \\
\text { e }\end{array}$ \\
Pelo menos três pontos nos seguintes critérios: \\
\hline Psoríase cutânea atual & 2 pontos \\
\hline História de psoríase & 1 ponto \\
\hline História familiar de psoríase & 1 ponto \\
\hline Dactilite & 1 ponto \\
\hline Neoformação óssea justa-articular & 1 ponto \\
\hline Fator reumatóide negativo & 1 ponto \\
\hline Distrofia ungueal & 1 ponto \\
\hline Extraída da referência 65. &
\end{tabular}

\section{TRATAMENTO}

A artrite psoriásica é multifatorial, envolvendo distintos aspectos que devem ser considerados na decisão do tratamento. É necessário individualizar a terapêutica levandose em conta o predomínio axial, periférico, as dactilites e entesites, o acometimento cutâneo e ungueal, além das co-morbidades e considerações econômicas e sociais ${ }^{(67)}$ (D). Dentre as doenças reumáticas auto-imunes, a artrite psoriásica é uma daquelas onde o estresse emocional representa um importante fator desencadeante da crise tanto articular quanto cutânea; sendo assim, o suporte psicológico é importante e indispensável na condução dos pacientes psoriásicos, principalmente para assegurar-lhes que a doença é controlável na maioria dos $\operatorname{casos}^{(68)}(\mathbf{D})$.

Os $A I N H s$ são utilizados de rotina no tratamento da artrite psoriásica, cursando com evidente melhora da dor articular, mas não são capazes de alterar sua evolução; devem ser utilizados de forma intermitente, enquanto houver atividade de doença. Não existe nenhum AINH específico comprovadamente melhor que os demais ${ }^{(69)}(\mathbf{D})$; no entanto, é importante salientar que o ácido acetilsalicílico, a indometacina e os oxicams podem exacerbar as lesões da pele ${ }^{(70)}(\mathbf{D})$. Quanto aos corticosteróides, a prednisona em doses baixas (até $10 \mathrm{mg} /$ dia, ou equivalente) pode eventualmente ser utilizada em casos de artrite periférica persistente; a retirada da droga deve ser lenta, a fim de se evitar rebote da doença ${ }^{(71)}(\mathbf{D})$. Não existem evidências para embasar o uso de altas doses de corticosteróides na artrite psoriásica.

Nos casos não responsivos aos AINHs, a droga de primeira escolha é o metotrexato, em doses habituais (por via oral ou intramuscular), até se obter controle da doença ${ }^{(72)}$ (B). A hepatotoxicidade do metotrexato na artrite psoriá- 
sica parece ser maior que a observada em pacientes com artrite reumatóide ${ }^{(73)}(\mathbf{C})$. A sulfasalazina, nas doses de 1,0 a 3,0 g/dia, demonstrou ser eficaz apenas com relação ao componente articular periférico da doença ${ }^{(74)}(\mathbf{A})$. A ciclosporina, nas doses de 3 a $5 \mathrm{mg} / \mathrm{kg} /$ dia, parece ser eficiente tanto para o componente articular quanto cutâneo ${ }^{(75)}(\mathbf{D})$, também apresentando boa resposta quando comparado ao uso do metotrexato ${ }^{(76)}(\mathbf{A})$ e da sulfasalazina ${ }^{(77)}(\mathbf{B})$. Estudo multicêntrico controlado recente mostrou que a combinação entre ciclosporina e metotrexato é uma boa alternativa nos pacientes parcialmente responsivos ao metotrexato ${ }^{(78)}$ (A). O uso da leflunomida, na dose de $20 \mathrm{mg} /$ dia, também tem mostrado bons resultados ${ }^{(79)}(\mathbf{A})$.

De forma semelhante à EA, os inibidores do TNF $\alpha$ apresentam resultados satisfatórios tanto na doença articular quanto cutânea, em adultos e crianças. Os agentes biológicos anti-TNF $\alpha$ aprovados para tratamento da artrite psoriásica, baseado em estudos controlados para avaliar eficácia e segurança, são o infliximabe, o etanercepte e o adalimumabe. O infliximabe tem mostrado boa resposta clínica $^{(80)}(\mathbf{A})$ e inibição da progressão radiológica ${ }^{81}(\mathbf{A})$ após um ano de tratamento. $\mathrm{O}$ etanercepte também tem demonstrado resposta clínica sustentada em um ano ${ }^{(82)}(\mathbf{A})$ e inibição da progressão radiológica após dois anos de $\mathrm{uso}^{(83)}(\mathbf{A})$.

\section{REFERÊNCIAS}

1. Moll JHM, Haslock I, MacRae I, et al.: Associations between ankylosing spondylitis, psoriatic arthritis, Reiter's disease, the intestinal arthropathies, and Behcet's syndrome. Medicine 53:343-64, 1974.

2. Braun J, Sieper J: Building consensus on nomenclature and disease classification for ankylosing spondylitis: results and discussion of a questionnaire prepared for the International Workshop on New Treatment Strategies in Ankylosing Spondylitis, Berlin, Germany, 18-19 January 2002. Ann Rheum Dis 61(Supl. III):61-7, 2002.

3. Dougados M, van der Linden S, Juhlin R, et al.: The European Spondylarthropathy Study Group preliminary criteria for the classification of spondylarthropathy. Arthritis Rheum 34:1218-27, 1991.

4. Van der Linden S, Van der Heijde D: Ankylosing spondylitis: clinical features. Rheum Dis Clin North Am 24:663-76, 1998.

5. Calin A, Elswood J, Rigg S, Skevington SM: Ankylosing spondylitis - An analytical review of 1,500 patients: the changing pattern of the disease. J Rheumatol 15:1234-8, 1988.

6. Reveille JD, Ball EJ, Khan MA: HLA-B27 and genetic predisposing factors in spondyloarthropathies. Curr Opin Rheumatol 13:265-72, 2001.

7. Sampaio-Barros PD, Bertolo MB, Kraemer MH, et al.: Primary ankylosing spondylitis: patterns of disease in a Brazilian population of 147 patients. J Rheumatol 28:560-5, 2001.
O adalimumabe também tem mostrado melhora clínica articular e cutânea sustentada após um ano de tratamento ${ }^{(84)}$ (A). Os agentes anti-TNF $\alpha$ na AP têm o potencial de levar a um alívio sintomático e auxiliar a prevenir a progressão da doença, com significativa melhora da qualidade de vida dos pacientes. Nenhum trabalho mostrou que um agente anti-TNF $\alpha$ específico é superior aos demais.

Após análise das recomendações para o uso de agentes biológicos nas espondiloartropatias, podemos ratificar os seguintes critérios: seu uso está indicado quando a terapia com pelo menos dois DMARDs não foi efetiva, por um prazo de pelo menos 6 meses. Deve ser considerada doença articular em atividade quando existe três ou mais articulações dolorosas e/ou edemaciadas, podendo ou não estar associado às dactilites e à doença cutânea em atividade. Indica-se a substituição ou troca de biológicos se não há resposta a um deles de maneira individual. Deve-se obedecer aos mesmos cuidados rigorosos quanto ao seguimento dos pacientes espondilíticos.

Estudos pilotos com outros agentes biológicos, como bloqueadores da ativação do linfócito T (CD2, alefacepte; CDlla, efalizumabe; CD80/CD86, abatacepte) e anticorpo monoclonal contra receptor de interleucina-6 (tocilizumabe), estão em andamento ${ }^{(85)}(\mathbf{D})$.

8. Wang SW, Davis JC Jr.: Clinical aspects of ankylosing spondylitis In: Weisman MH, Reveille JD, van der Heijde D (eds). Ankylosing spondylitis and the spondyloarthropathies. Philadelphia: Mosby Elsevier, 2006;145-53.

9. Van der Linden S, Valkenburg HA, Cats A: Evaluation of diagnostic criteria for ankylosing spondylitis: a proposal for modification of the New York criteria. Arthritis Rheum 27:361-8, 1984.

10. Van der Heijde D, Landewe R: Assessment of disease activity, function, and quality of life. In: Weisman MH, Reveille JD, van der Heijde D (eds). Ankylosing spondylitis and the spondyloarthropathies. Philadelphia: Mosby Elsevier, 2006; 206-13.

11. Braun J, Baraliakos X: Imaging in ankylosing spondylitis. In: Weisman MH, Reveille JD, van der Heijde D (eds). Ankylosing spondylitis and the spondyloarthropathies. Philadelphia: Mosby Elsevier, 2006;187-204.

12. Garrett S, Jenkinson T, Kennedy LG, Whitelock H, Gaisford P, Calin A: A new approach to defining disease status in ankylosing spondylitis: the Bath Ankylosing Spondylitis Disease Activity Index. J Rheumatol 21:2286-91, 1994.

13. Cusmanich KG: Validação para a língua portuguesa dos instrumentos de avaliação de índice funcional e índice de atividade de doença em pacientes com espondilite anquilosante. Dissertação de Mestrado em Ciências, Faculdade de Medicina da Universidade de São Paulo. Banco de Tese Capes, 2006.

14. Calin A, Garrett S, Whitelock $\mathrm{H}$, et al.: A new approach to defining functional ability in ankylosing spondylitis: the development of the Bath Ankylosing Spondylitis Functional Index. J Rheumatol 21:2281-5, 1994. 
15. Shinjo SK, Goncalves R, Kowalski S, Goncalves CR: BrazilianPortuguese version of the Health Assessment Questionnaire for Spondyloarthropathies (HAQ-S) in patients with ankylosing spondylitis: a translation, cross-cultural adaptation, and validation. Clin Rheumatol 2007; 26:1254-8.

16. Dagfinrud H, Kvien TK, Hagen KB: The Cochrane review of physiotherapy interventions for ankylosing spondylitis. J Rheumatol 32:1899-906, 2005.

17. Clegg DO: Treatment of ankylosing spondylitis. J Rheumatol 33(Supl. 78):24-31, 2006.

18. Van der Heijde D, Baraf HS, Ramos-Remus C et al.: Evaluation of the efficacy of etoricoxib in ankylosing spondylitis: results of a fifty-two-week, randomized, controlled study. Arthritis Rheum 52:1205-15, 2005.

19. Barkhuizen A, Steinfeld S, Robbins J, West C, Coombs J, Zwillich $S$ : Celecoxib is efficacious and well tolerated in treating signs and symptoms of ankylosing spondylitis. J Rheumatol 33:1805-12, 2006.

20. Wanders A, Van der Heijde D, Landewé R, et al.: Nonsteroidal antiinflammatory drugs reduce radiographic progression in patients with ankylosing spondylitis: a randomized clinical trial. Arthritis Rheum 52:1756-65, 2005.

21. Ward MM: Prospects for disease modification in ankylosing spondylitis: do nonsteroidal antiinflammatory drugs do more than treat symptoms? Arthritis Rheum 52:1634-6, 2005.

22. Dougados M, Dijkmans B, Khan M, Maksymowych W, Van der Linden S, Brandt J: Conventional treatments for ankylosing spondylitis. Ann Rheum Dis 61(Supl. III):40-50, 2002.

23. Peters ND, Ejstrup L: Intravenous methylprednisolone pulse therapy in ankylosing spondylitis. Scand J Rheumatol 21:134-8, 1992.

24. Braun J, Bollow M, Seyrekbasan F, et al.: Computed tomography guided corticosteroid injection of sacroiliac joint in patients with spondyloarthropathy with sacroiliitis: clinical outcome and followup by dynamic magnetic resonance imaging. J Rheumatol 23:659-64, 1996.

25. Gratacós J, Collado A, Pons F, et al.: Significant loss of bone mass in patients with early, active ankylosing spondylitis: a followup study. Arthritis Rheum 42:2319-24, 1999.

26. Mitra D, Elvins DM, Speden DJ, Collins AJ: Prevalence of vertebral compression fractures in mild ankylosing spondylitis and their relationship to bone mineral density. Rheumatology 39:85-9, 2000.

27. Zochling J, Van der Heijde D, Burgos-Vargas R, et al.: ASAS/ EULAR recommendations for the management of ankylosing spondylitis. Ann Rheum Dis 65:442-52, 2006.

28. Dougados $M$, van der Linden S, Leirisalo-Repo M, et al.: Sulfasalazine in the treatment of spondylarthropathy: a randomized multicenter, double-blind, placebo-controlled study. Arthritis Rheum 38:618-27, 1995.

29. Benitez-del-Castilho JM, Garcia-Sanchez J, Iradier T, Bañares A: Sulfasalazine in the prevention of anterior uveitis associated with ankylosing spondylitis. Eye 14:340-3, 2000.

30. Chen J, Liu C: Sulfasalazine for ankylosing spondylitis. Cochrane Database Syst Rev 18(2): CD004800, 2005.

31. Sampaio-Barros PD, Costallat LTL, Bertolo MB, Marques Neto JF, Samara AM: Methotrexate in the treatment of ankylosing spondylitis. Scand J Rheumatol 29:160-2, 2000.

32. Chen J, Liu C, Lin J: Methotrexate for ankylosing spondylitis. Cochrane Database Syst Rev 18(4):CD004524, 2006.
33. Akkoc N, van der Linden S, Khan MA: Ankylosing spondylitis and symptom-modifying vs. disease modifying therapy. Best Pract Res Clin Rheumatol 20:539-57, 2006.

34. Huang F, Gu J, Zhao W, Zhu J, Zhang J, Yu DTY: One year open label trial of thalidomide in ankylosing spondylitis. Arthritis Rheum 47:249-54, 2002.

35. Maksymowych WP, Jhangri GS, Fitzgerald A, et al.: A sixmonth randomized, controlled, double-blind, dose-response comparison of intravenous pamidronate $(60 \mathrm{mg}$ versus $10 \mathrm{mg})$ in the treatment of nonsteroidal anti-inflammatory drug-refractory ankylosing spondylitis. Arthritis Rheum 46:766-73, 2002.

36. Van den Bosch F, Kruithof E, Baeten D, et al.: Randomized double-blind comparison of chimeric monoclonal antibody to tumor necrosis factor $\alpha$ (infliximab) versus placebo in active spondylarthropathy. Arthritis Rheum 46:755-65, 2002.

37. Kruithof E, Van den Bosch F, Baeten D, et al.: Repeated infusions of infliximab, a chimeric anti-TNF $\alpha$ monoclonal antibody, in patients with active spondyloarthropathy: one year follow up. Ann Rheum Dis 61:207-12, 2002.

38. Braun J, Landewe R, Hermann K-G, et al.: Major reduction in spinal inflammation in patients with ankylosing spondylitis after treatment with infliximab: results of a multicenter, randomized, double-blind, placebo-controlled magnetic resonance imaging study. Arthritis Rheum 54:1646-52, 2006.

39. Braun J, Brandt J, Listing J, et al.: Treatment of ankylosing spondylitis with infliximab: a randomized controlled multicentre trial. Lancet 359:1187-93, 2002.

40. Braun J, Brandt J, Listing J, et al.: Long-term efficacy and safety of infliximab in the treatment of ankylosing spondylitis. Arthritis Rheum 48:2224-33, 2003.

41. Braun J, Baraliakos X, Listing J, Sieper J: Decreased incidence of anterior uveitis in patients with ankylosing spondylitis treated with the anti-tumor necrosis factor agents infliximab and etanercept. Arthritis Rheum 52:2447-51, 2005.

42. Braun J, Baraliakos X, Brandt J, et al.: Persistent clinical response to the anti-TNF- $\alpha$ antibody infliximab in patients with ankylosing spondylitis over 3 years. Rheumatology 44:670-6, 2005.

43. Baraliakos X, Listing J, Brandt J, et al.: Clinical response to discontinuation of anti-TNF therapy in patients with ankylosing spondylitis after 3 years of continuous treatment with infliximab. Arthritis Res Ther 7:R439-44, 2005.

44. Baraliakos X, Listing J, Rudwaleit M, et al.: Safety and efficacy of readministration of infliximab after long-term continuous therapy and withdrawal in patients with ankylosing spondylitis. J Rheumatol 34:510-5, 2007.

45. Brandt J, Khariouzov A, Listing J, et al.: Six-month results of a double-blind, placebo-controlled trial of etanercept treatment in patients with active ankylosing spondylitis. Arthritis Rheum 48:1667-75, 2003.

46. Davis Jr. JC, van der Heijde D, Braun J, et al.: Recombinant human tumor necrosis factor receptor (etanercept) for treating ankylosing spondylitis: a randomized controlled trial. Arthritis Rheum 48:3230-6, 2003.

47. Maksymowych WP, Poole AR, Hiebert L, et al.: Etanercept exerts beneficial effects on articular cartilage biomarkers of degradation and turnover in patients with ankylosing spondylitis. J Rheumatol 32;1911-7, 2005.

48. Baraliakos X, Brandt J, Listing J, et al.: Outcome of patients with active ankylosing spondylitis after two years of therapy with etanercept: clinical and magnetic resonance imaging data. Arthritis Rheum 53:856-63, 2005. 
49. Davis JC, Van der Heijde D, Braun J, et al.: Sustained durability and tolerability of etanercept in ankylosing spondylitis for 96 weeks. Ann Rheum Dis 64;1557-62, 2005.

50. Van der Heijde D, Da Silva JC, Dougados M, et al.: Etanercept $50 \mathrm{mg}$ once weekly is as effective as $25 \mathrm{mg}$ twice weekly in patients with ankylosing spondylitis. Ann Rheum Dis 65:1572-7, 2006.

51. Van der Heijde D, Kivitz A, Schiff MH, et al.: Efficacy and safety of adalimumab in patients with ankylosing spondylitis. Arthritis Rheum 54;2136-46, 2006.

52. Haibel H, Rudwaleit M, Brandt HC, et al.: Adalimumab reduces spinal symptoms in active ankylosing spondylitis: clinical and magnetic resonance imaging results of a fifty-two-week openlabel trial. Arthritis Rheum 54:678-81, 2006.

53. Braun J, Davis J, Dougados M, et al.: First update of the international ASAS consensus statement for the use of anti-TNF agents in patients with ankylosing spondylitis. Ann Rheum Dis 65:316-20, 2006.

54. Carmona L, Gomez-Reino JJ, on behalf of the BIOBADASER Group: Survival of TNF antagonists in spondylarthritis is better than in rheumatoid arthritis. Data from the Spanish registry BIOBADASER. Arthritis Res Ther R72, 2006.

55. Rudwaleit M, Listing J, Brandt J, Braun J, Sieper J: Prediction of a major clinical response (BASDAI 50) to tumour necrosis factor $\alpha$ blockers in ankylosing spondylitis. Ann Rheum Dis 63:665-70, 2004.

56. Delaunay C, Farrenq V, Marini-Portugal A, et al.: Infliximab to etanercept switch in patients with spondyloarthropathies and psoriatic arthritis: preliminary data. J Rheumatol 32:2183-5, 2005.

57. Moll JMH, Wright V: Psoriatic arthritis. Semin Arthritis Rheum 3:55-78, 1973.

58. Gladman DD, Antoni C, Mease P, Clegg DO, Nash P: Psoriatic arthritis: epidemiology, clinical features, course, and outcome. Ann Rheum Dis 64(Supl. II):iil4-iil7, 2005.

59. Griffiths CEM, Camp RDR, Barker JN: Psoriasis. In: Champion RH, Burton JL, Burns DA, et al. Rook/Wilkinson/Ebling Textbook of dermatology. 7a ed. Oxford: Blackwell Science, 2004;35.1-65.

60. Espinoza LR, Cuellar ML: Psoriatic arthritis and spondylitis: a clinical approach. In Calin A, Taurog JD (editors). Spondylarthritides. Oxford: Oxford University Press, 1998:97-111.

61. Mease P, Goffe BS: Diagnosis and treatment of psoriatic arthritis. J Am Acad Dermatol 52:1-19, 2005.

62. Balding J, Kane D, Livingstone W, et al.: Cytokine gene polymorphisms: Association with psoriatic arthritis susceptibility and severity. Arthritis Rheum 48:1408-13, 2003.

63. Sibilia J: Psoriasis: skin and joints, same fight? JEADV 20(Supl. 2):56-72, 2006.

64. Veale D, Ritchlin C, Fitz Gerald O: Immunopathology of psoriasis and psoriatic arthritis. Ann Rheum Dis 65:26-9, 2005.

65. Taylor W, Gladman D, Helliwell P, et al.: Classification criteria for psoriatic arthritis: development of new criteria from a large international study. Arthritis Rheum 54:2665-73, 2006.

66. Khan M-F, Chamot A-M: SAPHO syndrome. Rheum Dis Clin North Am 18:225-46, 1992.

67. Kavanaugh AF, Ritchlin CT, and the GRAPPA Treatment Guideline Committee: Systematic review of treatment for psoriatic arthritis: an evidence based approach and basis for treatment guidelines. J Rheumatol 33:1417-21, 2006.
68. Greaves MW, Weinstein GD: Treatment of psoriasis. New Engl J Med 332:581-8, 1995.

69. Gladman DD: Effectiveness of psoriatic arthritis therapies. Semin Arthritis Rheum 33:29-37, 2003.

70. Tsankov N, Kazandjieva J, Drenovska K: Drugs in exacerbation and provocation of psoriasis. Clin Dermatol 16:333-51, 1998.

71. Soriano ER, McHugH NJ: Therapies for peripheral joint disease in psoriatic arthritis: a systematic review. J Rheumatol 33:1422-30, 2006.

72. Espinoza LR, Zakraoui I, Espinoza CG, et al.: Psoriatic arthritis: clinical response and side effects to methotrexate therapy. J Rheumatol 19:872-7, 1992.

73. Tilling L, Townsend S, David J: Methotrexate and hepatic toxicity in rheumatoid arthritis and psoriatic arthritis. Clin Drug Investig 26:55-62, 2006.

74. Clegg DO, Reda RJ, Mejias E, et al.: Comparison of sulfasalazine and placebo in the treatment of psoriatic arthritis: a Department of Veterans Affairs Cooperative Study. Arthritis Rheum 39:2013-20, 1996.

75. Olivieri I, Salvarani C, Cantini F, et al.: Therapy with cyclosporine in psoriatic arthritis. Semin Arthritis Rheum 27:36-43, 1997.

76. Spadaro A, Riccieri V, Silli-Scavalli A, Sensis F, Taccari E, Zoppini A: Comparison of cyclosporin A and methotrexate in the treatment of psoriatic arthritis: a one-year prospective study. Clin Exp Rheumatol 13:589-93, 1995.

77. Salvarani C, Macchioni P, Olivieri I, et al.: A comparison of cyclosporine, sulfasalazine, and symptomatic therapy in the treatment of psoriatic arthritis. J Rheumatol 28;2274-82, 2001.

78. Fraser AD, van Kujik AWR, Westhovens R, et al.: A randomised, double-blind, placebo-controlled, multicentre trial of combination therapy with methotrexate plus ciclosporin in patients with active psoriatic arthritis. Ann Rheum Dis 64:859-64, 2005.

79. Kaltwasser JP, Nash P, Gladman D, et al.: Efficacy and safety of leflunomide in the treatment of psoriatic arthritis and psoriasis: a multinational, double-blind, randomized, placebo-controlled clinical trial. Arthritis Rheum 50:1939-50, 2004.

80. Kavanaugh A, Krueger GG, Beutler A, et al.: Infliximab maintains a high degree of clinical response in patients with active psoriatic arthritis through 1 year of treatment: results from the IMPACT 2 trial. Ann Rheum Dis 66:498-505, 2007.

81. Kavanaugh A, Antoni CE, Gladman D, et al.: The Infliximab Multinational Psoriatic Arthritis Controlled Trial (IMPACT): results of radiographic analyses after 1 year. Ann Rheum Dis 65:1038-43, 2006.

82. Mease PJ, Kivitz AJ, Burch FX, et al.: Etanercept treatment of psoriatic arthritis: safety, efficacy, and effect on disease progression. Arthritis Rheum 50:2264-72, 2004.

83. Mease PJ, Kivitz AJ, Burch FX, et al.: Continued inhibition of radiographic progression in patients with psoriatic arthritis following 2 years of treatment with etanercept. J Rheumatol 33:712-21, 2006.

84. Gladman DD, Mease PJ, Ritchlin CT, et al.: Adalimumab for long-term treatment of psoriatic arthritis: forty-eight week data from the adalimumab effectiveness in psoriatic arthritis trial. Arthritis Rheum 56:476-88, 2007.

85. Turkiewicz AM, Moreland LW: Psoriatic arthritis: current concepts on pathogenesis-oriented therapeutic options. Arthritis Rheum 56:1051-66, 2007. 